\title{
Design Patterns for Math Problems and Learning Support in Online Learning Systems
}

\author{
PAUL SALVADOR INVENTADO \& PETER SCUPELLI School of Design, Carnegie Mellon University
}

\begin{abstract}
Increasingly, many institutions and students benefit from online learning systems each year. For example, in 2016 Massive Open Online Courses (MOOCS) reported as many as 16 million enrolled students and online tutoring systems reported over half a million enrolled students. In the literature, many design patterns capture online learning system designs for class management, discussion facilitation, lecture delivery, and feedback. In this paper, we describe design patterns that describe finer-grained activities within online learning systems such as the design of problem-solving activities and their associated learning support. The three patterns presented in this paper describe designs for constructing math-problem content and corresponding learning support for students who answer these problems Mastery Learning Templates, Explain Worked Solutions, and Scaffold Problems with Guide Questions. We uncovered these patterns using the data-driven design pattern production (3D2P) methodology on data collected from the ASSISTments online learning system. The design patterns we describe were mined from data on student interactions with an online learning system and were linked to existing learning-science literature.
\end{abstract}

• Applied computing Distance learning • Human-centered computing User centered design

Additional Key Words and Phrases: Design patterns, online learning systems, learning support, problem solving, data driven design pattern production, ASSISTments

\section{ACM Reference Format:}

Paul Salvador Inventado and Peter Scupelli. 2016. Design Patterns for Math Problems and Learning Support in Online Learning Systems. VikingPLoP '16. (April 07-10 2016), 16 pages

DOI: http://dx.doi.org/10.1145/3022636.3022644

\section{INTRODUCTION}

Online learning systems, such as ASSISTments and Cognitive Tutor, have been used successfully to facilitate students' learning of Math content (Heffernan \& Heffernan 2014, U.S. Dept. of Education 2009). These systems help students practice solving math problems and also provide them with appropriate learning support when necessary (e.g., hints, scaffolds, related content). Currently, ASSISTments has over 300,000 users and Cognitive Tutor has over 500,000 users (Heffernan \& Heffernan 2014, U.S. Dept. of Education 2009). The effectiveness of these systems and the number of users who benefit from them emphasize the impact of online learning systems on math education and the need to ensure the quality of such systems.

Existing online-learning-system design patterns often describe higher-level designs. For example, the provision of math problem solving exercises implement the Try It Yourself design pattern and corresponding learning support for the problems implement the Build and Maintain Confidence design pattern (Bergin et al. 2012). However, there are fewer design patterns that describe the construction of problems and their corresponding learning support.

This paper presents three patterns: Mastery Learning Templates, Worked Solutions, and Scaffold Problems with Guide Questions. The data-driven design pattern production (3D2P) methodology was used to uncover these patterns from data describing students' interaction with math problems and associated learning support in an online learning system called ASSISTments. Teachers, content creators, and system developers may use these design patterns to help them construct high quality math problems and corresponding learning support for online learning systems.

The following sections provide a review of related literature, a brief discussion about the methodology used to uncover the patterns presented in the paper, the design patterns, and future work.

Permission to make digital or hard copies of all or part of this work for personal or classroom use is granted without fee provided that copies are not made or distributed for profit or commercial advantage and that copies bear this notice and the full citation on the first page. Copyrights for components of this work owned by others than the author(s) must be honored. Abstracting with credit is permitted. To copy otherwise, or republish, to post on servers or to redistribute to lists, requires prior specific permission and/or a fee. Request permissions from Permissions@acm.org.

VikingPLoP '16, April 07 - 10, 2016, Leerdam, AA, Netherlands

Copyright is held by the owner/author(s). Publication rights licensed to ACM.

ACM 978-1-4503-4200-1/16/04 $\ldots 15.00$

DOI: http://dx.doi.org/10.1145/3022636.3022644 


\section{RELATED LITERATURE}

Pedagogical design patterns may be used in the design of math problem content and learning support in online learning systems. For example, Köppe and colleagues' (2015) in-class meeting design patterns for flipped classrooms help to ensure that students get necessary support in deepening understanding of topics discussed and to correct possible misconceptions. The Use Student Solutions design pattern encourages the use of students' submitted solutions to identify common misconceptions or effective solutions. These findings may be used to design problem or learning support content in online learning systems that may be more meaningful and useful for students.

Warburton and Mor are compiling a Pattern Language for MOOCs to address various challenges in online learning systems such as those involving course content, individual differences, and failure points in technology (Mor \& Warburton 2015, Warburton \& Mor 2015). Some of these patterns may also be used in online learning systems such as the Six Minute Video design pattern that suggests the use of short videos to keep student attention. This can be used to guide the creation of videos for math-problem content or its corresponding learning support.

Bergin et al.'s (2012) pedagogical patterns are quite robust and can be used in most educational contexts. The patterns address various problems on active learning, feedback, and experiential learning to name a few. Pedagogical patterns such as Different Exercise Levels, Feedback Sandwich, and Differentiated Feedback, are particularly interesting because they can be used to select and arrange math-problem content, to provide encouraging learning support, and to personalize feedback according to each student's needs.

Many pedagogical design patterns promote practice problems and learning support at a higher level, but there are fewer patterns that describe the proper design of actual problem and learning support content, which are essential for online learning systems.

\section{DATA-DRIVEN DESIGN PATTERN PRODUCTION}

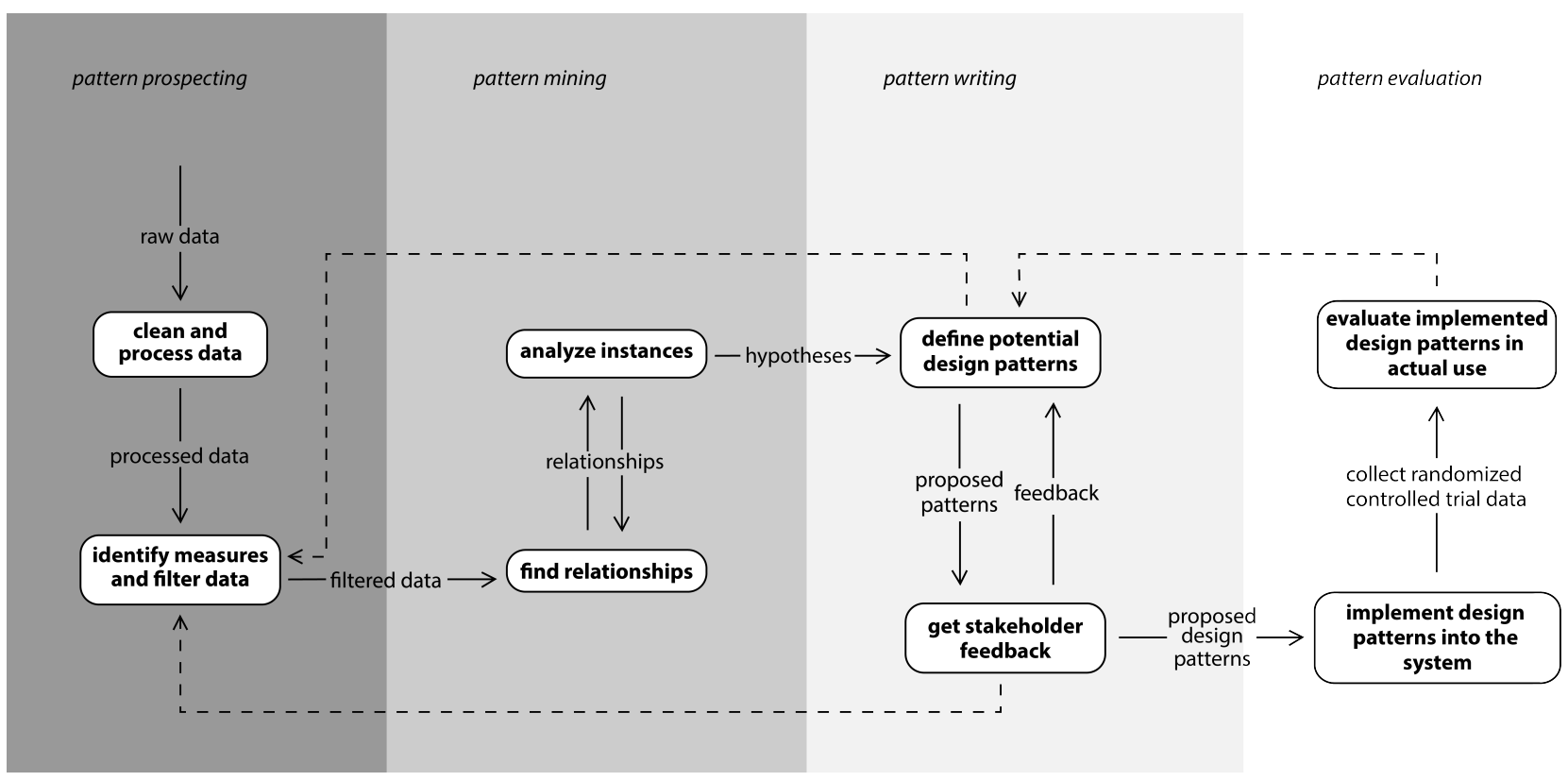

Fig. 1. Data-driven design pattern production methodology (Image courtesy of the Learning Environments Lab).

The design patterns presented in this paper were uncovered using the data-driven design pattern production (3D2P) methodology. 3D2P is a four-step iterative process used to uncover design patterns from collected data (Inventado \& Scupelli 2015a). As illustrated in Figure 1, 3D2P starts with pattern prospecting to find interesting relationships in the data. These relationships are investigated further in the pattern-mining step to develop hypotheses based on recurring problems and high quality solutions uncovered. Literature and experts in the field are consulted to confirm the validity of the hypotheses. Validated hypotheses are used to write proposed patterns and are refined through shepherding, writing workshops, or discussions with experts and stakeholders. Refined design patterns are then implemented in existing systems and evaluated to measure performance. Randomized controlled trials (RCTs) are conducted to compare the resulting outcome measures 
when a design pattern was applied or not (e.g., students' learning gain, level of engagement, time on task). Results of the evaluation are used to further refine the design pattern as needed.

3D2P differs from other pattern mining methods in that it uses data about interactions with existing design implementations to uncover recurring problems and high quality solutions in the domain of interest. The role of pattern authors and experts is to interpret and verify uncovered patterns from the data, distinguish high quality patterns, and express validated patterns into design patterns. Some uncovered patterns may be irrelevant, which the pattern authors may reject while validated patterns may be further refined. These patterns can be used to enhance the quality of the system from which the data was collected, to adapt quality solutions into other systems that use similar design implementations, or to design new systems.

The patterns uncovered through the methodology are limited by what is available in the data. It is possible that some recurring problems and high quality solutions in the domain are not uncovered because they were not captured in the data used during analysis. Easily collected big data is preferred when applying the 3D2P methodology so that more patterns may be uncovered.

\section{DESIGN PATTERNS}
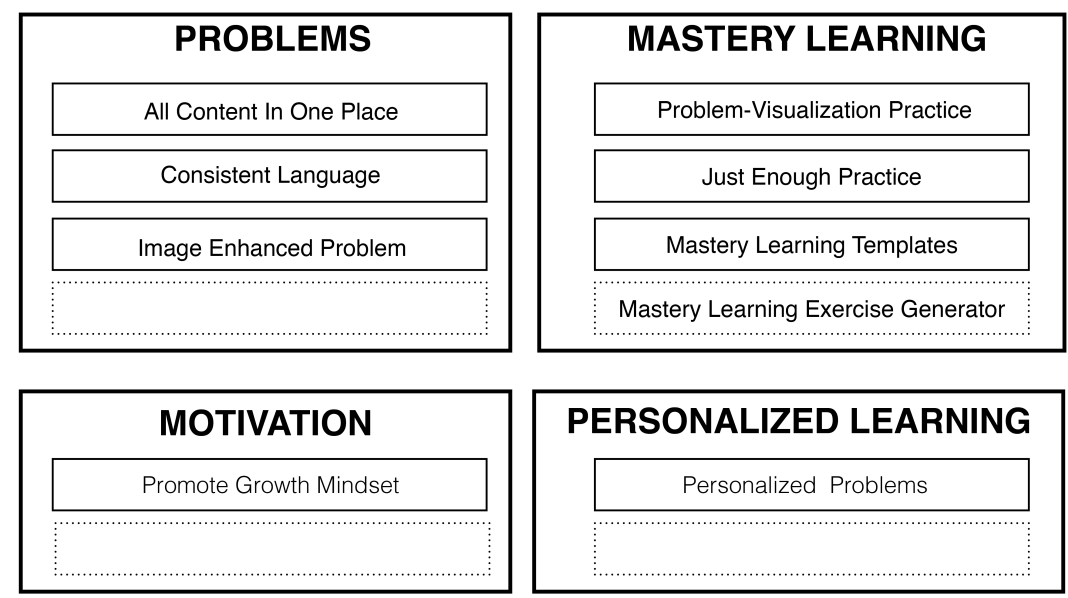

\section{PERSONALIZED LEARNING}
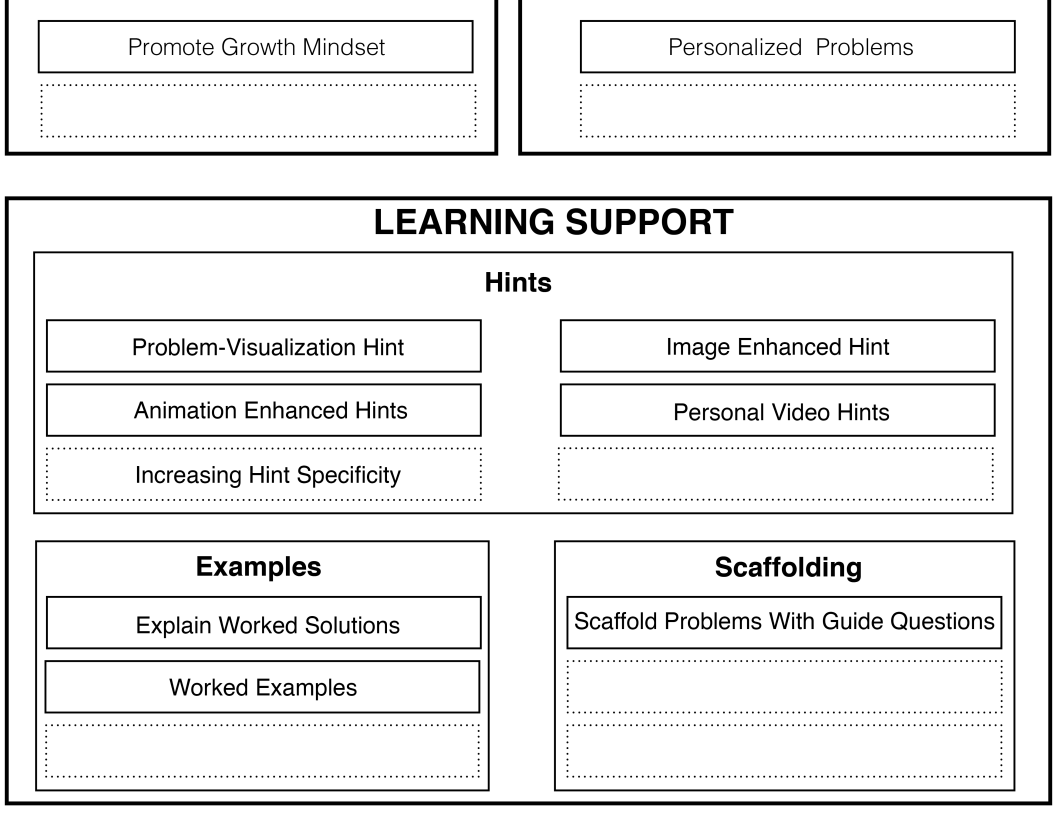

Fig. 2. Visualization of the Pattern Language for Math problems and Learning Support in Online Learning Systems (Image courtesy of the Learning Environments Lab).

The patterns presented in this paper are part of a pattern language currently being compiled by the authors for math online learning systems, which is illustrated in Figure 2. The innermost rectangles represent design patterns. Published patterns are drawn using solid lines and patterns-in-development are drawn with dotted lines. Empty rectangles indicate that other patterns may later be included in the language. The design patterns are grouped into five categories represented by outer rectangles that enclose the patterns: Problems, Learning Support, Personalized Learning, Motivation, and Mastery Learning. Problems refer to patterns that deal with the 
design of math problem content. Learning Support refers to patterns that deal with the design of math learningsupport content, how they are presented, or how much learning support should be provided. It is further categorized according to the type of learning support provided such as Hints, Examples, and Scaffolding. Personalized Learning refers to patterns that deal with designs for adapting problem content or learningsupport content so that it is more appropriate to the student's background knowledge, affective state, personality, and so forth. Motivation refers to patterns that deal with designs for maintaining student motivation while maximizing learning. Finally, Mastery Learning refers to patterns that deal with the design of math problems or problem sets to promote skill mastery. Some of these design patterns have recently been published, which can be found in Inventado and Scupelli (2015a), Inventado and Scupelli (2015b), Inventado and Scupelli (2016a), and Inventado and Scupelli (2016b).

The design pattern format used in this paper differs from other formats in two ways: the addition of in-text citations, and the addition of an evidences section. When appropriate, in-text citations were used for linking forces, consequences, and evidence to pre-existing literature. Such references allow the reader to learn more about the statement in question. The evidence section is similar to the "Known uses" section, but emphasizes supporting theories, empirical data, and known implementations of the pattern. The patterns presented in this paper for example, show empirical data uncovered from the 3D2P methodology as evidence. Evidences justify the recurrence of the problem and the effectiveness of the pattern's solution.

Table 1 provides summaries of the three patterns presented in the following subsections. Table 2 provides summaries of design patterns referenced in the paper.

Table 1. Learning-support design patterns for problem-solving activities in online learning systems

\begin{tabular}{|l|l|}
\hline Pattern name & Summary \\
\hline Mastery Learning Templates & $\begin{array}{l}\text { Create templates that can be used to generate problems and corresponding } \\
\text { hints for mastery learning exercises instead of encoding problem and hint } \\
\text { variations manually. }\end{array}$ \\
\hline Explain Worked Solutions & $\begin{array}{l}\text { Provide students with clearly explained worked solutions when they are } \\
\text { unable to answer problems correctly despite receiving support. }\end{array}$ \\
\hline Scaffold Problems with Guide Questions & $\begin{array}{l}\text { Ask students to answer guide questions that may help them recall prior } \\
\text { knowledge or make inferences necessary to solve a problem. }\end{array}$ \\
\hline
\end{tabular}

Table 2. Referenced Patterns

\begin{tabular}{|l|l|}
\hline Pattern Name & Summary \\
\hline $\begin{array}{l}\text { Build and Maintain Confidence } \\
\text { (Bergin et al. 2012) }\end{array}$ & $\begin{array}{l}\text { Give students problem-solving activities with appropriate feedback so they } \\
\text { may apply what they learned, discover that there are multiple solutions, and } \\
\text { realize they can implement solutions themselves. }\end{array}$ \\
\hline $\begin{array}{l}\text { Digestible Packets } \\
\text { (Bergin et al. 2012) }\end{array}$ & $\begin{array}{l}\text { Reorganize long and complex topics into multiple topics that are short, } \\
\text { understandable, and useable. }\end{array}$ \\
\hline $\begin{array}{l}\text { Image-Enhanced Hint } \\
\text { (Inventado \& Scupelli 2016a) }\end{array}$ & $\begin{array}{l}\text { Clarify hints for math problems by adding images that disambiguate } \\
\text { confusing terms and explanations. }\end{array}$ \\
\hline $\begin{array}{l}\text { Increasing Hint Specificity } \\
\text { Inventado \& Scupelli under development) }\end{array}$ & $\begin{array}{l}\text { Allow students to sequentially request for hints, which reveal more specific } \\
\text { information as hints are requested. }\end{array}$ \\
\hline $\begin{array}{l}\text { Keep It Simple } \\
\text { (Cunningham \& Cunningham 2014) }\end{array}$ & $\begin{array}{l}\text { Designs should be kept as simple as possible while ensuring it achieves its } \\
\text { purpose. }\end{array}$ \\
\hline $\begin{array}{l}\text { Mastery Learning Exercise Generator } \\
\text { Inventado \& Scupelli under development) }\end{array}$ & $\begin{array}{l}\text { Generate and assign problem variations that test a particular skill to help } \\
\text { students master that skill. }\end{array}$ \\
\hline $\begin{array}{l}\text { One Concept - Several Implementations } \\
\text { (Bergin et al. 2012) }\end{array}$ & $\begin{array}{l}\text { Present and compare several implementations of an abstract concept so } \\
\text { learners understand its essence and not just associate it with a single } \\
\text { implementation. }\end{array}$ \\
\hline $\begin{array}{l}\text { Personalized Problems } \\
\text { (Inventado \& Scupelli 2015b) }\end{array}$ & $\begin{array}{l}\text { Assign problem-solving activities that are appropriate to a student's skill } \\
\text { level. }\end{array}$ \\
\hline $\begin{array}{l}\text { Try It Yourself } \\
\text { (Bergin et al. 2012) }\end{array}$ & $\begin{array}{l}\text { Ask students to answer exercises that will deepen their understanding of the } \\
\text { topic. }\end{array}$ \\
\hline $\begin{array}{l}\text { Worked Example } \\
\text { (Inventado \& Scupelli 2015b) }\end{array}$ & $\begin{array}{l}\text { When learners request for help to solve a problem, show them the complete } \\
\text { solution for a similar problem so they can learn from it and apply the } \\
\text { solution on the current problem. }\end{array}$ \\
\hline
\end{tabular}




\section{Mastery Learning Templates}

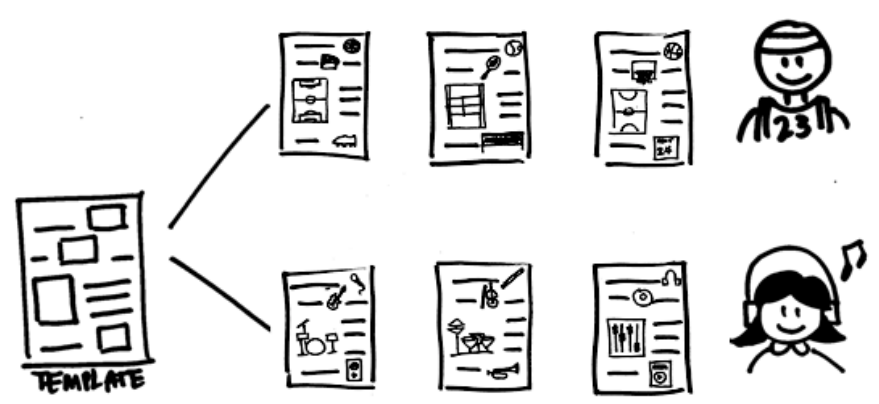

Context: An online learning system for math is used to help students master a skill they recently learned. One way to implement mastery learning (Bloom 1968, Guskey 2008, Kulik et al. 1990) is through problem-solving exercises that target a particular skill or set of skills. Students need to answer the exercises until they achieve a particular level of performance that equates to mastery (e.g., answer three problems correctly in a row, answer $90 \%$ of the problems correctly, answer all problems correctly within a given time limit). Another important aspect of mastery learning is learning support, such as hints, to help students remember key concepts, identify their mistakes, or make inferences (Hume et al. 1996). Learning support may help students solve the current problem or other related problems and achieve mastery.

Problem: Encoding problem and learning support content for mastery learning exercises in a math online learning system is tedious and prone to errors.

\section{Forces:}

1. Problem variations. Problems designed to test the same skill are often very similar, but are tedious to replicate (e.g., Problem: Simplify 6/21; Answer: 2/7 vs. Problem: Simplify 9/24; Answer: 3/8 vs. Problem: Simplify 15/30; Answer: 1/2).

2. Learning support variations. Learning support associated with problem variations are also similar and tedious to replicate (e.g., Hint: What number divides both 6 and 21? vs. Hint: What number divides both 9 and 24? vs. Hint: What number divides both 15 and 30?).

3. Replication error. Content creators may use "shortcuts" to save time and effort to encode similar content into a system. For example, content is copied and pasted then edited to produce different variations. Creating subtle variations may be confusing and could lead to errors such as duplicate variations, missing variations, unsuspected deletion of content, or unsuspected addition of content.

Solution: Therefore, create templates that can be used to generate problem and learning support variations. A template contains the content, but with variations replaced by placeholders. For example - Problem: Simplify $\% v\{a\} / \% v\{b\}$; Answer: $\% v\{$ answer\}; Hint: What number divides both $\% v\{a\}$ and $\% v\{b\}$. In this particular example, $\% v\{a\}$ is a placeholder for variable $a$ that is later populated by a content generator such as what is described in the Mastery Learning Exercise Generator design pattern (see Figures 3-5). Variables are merged with templates to produce problem variations. The example shown in the forces section for example, can be generated by configuring variable $a$ to contain values that are multiples of three, greater than three, and less than or equal to 15 (i.e., $6,9,12,15$ ), and variable $b$ to contain values that are multiples of three, greater than 20, and less than or equal to 30 (i.e., 21, 24, 27, 30). Answers can be stored in variables as well (i.e., $2 / 7,3 / 8,4 / 9,1 / 2$ ).

\section{Consequences:}

Benefits:

1. Content creators only need to create problem templates and specify placeholder values instead of encoding each problem variation.

2. Content creators can create learning support templates with associated placeholder values instead of encoding each variation. 
3. Encoding templates and identifying variable values separately require less cognitive load compared to encoding every variation. The reduction of cognitive load and frequency of encoding content may help content creators avoid mistakes (Head \& Helton 2014, Chapanis et al. 1949).

\section{Liabilities:}

1. The online learning system will need to support templates and problem generation.

2. Teachers and content creators need to learn how to use templates and other modules needed to automatically generate content.

3. Teachers and content creators need to carefully select variable values to avoid unintended problem or learning support variations. For example, content creators need to ensure that zeroes are not generated in the denominator of fraction values.

4. Creating a template is more difficult than encoding a problem. It is only worthwhile to create templates when there are many problem variations, which are tedious to encode and prone to errors.

5. Individual differences and learning contexts may affect the effectiveness of math problems and their associated learning support (Bjork 1994, Cen et al. 2007, Inventado et al. 2016, Salden et al. 2010). Templates may need to be generated for specific student populations.

\section{Evidence:}

\section{Supporting Theories}

1. Mastery learning is a method of instruction that encourages student mastery of prerequisite knowledge before moving on to a subsequent topic (Bloom 1968). It involves repeated learning support and testing until mastery of the topic is reached. Plenty of research has been conducted on mastery learning since it was introduced by Bloom. There have been debates about its effectiveness as a method of instruction, but most research findings still conclude that mastery learning has positive effects on student achievement and attitude (cf. Cox (1979), Guskey (2008), Guskey \& Gates (1985), Kulik et al. (1990), Slavin (1987)).

2. Errors in man-machine systems may be attributed to system issues or to human error (Chapanis et al. 1949). Errors may either be constant - average difference between actual outcomes and expected outcomes; or variable - variance between actual outcomes. Constant errors are often easier to correct because the system can be re-calibrated. Variable errors on the other hand require additional support to keep actions more consistent (e.g., training, immediate feedback, task simplification).

3. Repetitive tasks, especially those that require more attention, lead to high cognitive load and increase the probability of commiting errors (Head \& Helton 2014).

4. Processing similar items in working memory can cause confusion that may lead to decrease in performance (Wickens et al. 2004).

\section{Empirical Data}

Content in the ASSISTments online learning system is largely contributed by content creators and teachers (Heffernan \& Heffernan 2014). ASSISTments has a commenting functionality that students and teachers can use to report issues with problems or hints in the system. According to an analysis conducted on the comments received, around $45 \%$ of the comments were helpful in addressing system issues (Razzaq et al. 2008). Other comments were either repetitions or unrelated to ASSISTments. One common issue reported is that some problems, answers, and hints did not match or were encoded incorrectly. Errors might have been introduced when content creators encoded problem variations and got confused with the input values.

\section{Known Implementations}

Templates are commonly used in learning systems for content generation. Systems may use different syntax for their templates and utilize different mechanisms for setting and assigning placeholder values, but it is still a preferred approach because of its simplicity. For example, Cognitive Tutor Algebra uses model tracing to analyze the correctness of each step in students' solution as they solve an algebra problem (Koedinger \& Aleven, 2007). An example of an incorrect step may be: failure to simplify an equation. The incorrect steps identified through model tracing are used to populate placeholder values in hint templates and to generate hints that are shown to students who request help. Various hints are generated with a template without requiring the content creator to make each one. 
Intelligent Teaching Assistant for Programming (ITAP) helps students learn to program in Python by asking them to solve programming problems and providing them hints that describe what they can do next to reach the correct solution (Rivers \& Koedinger, 2015). ITAP uses a data-driven approach, which utilizes teachers' exemplar solutions and previously submitted student solutions to build a solution space. The most similar program state in the solution space is used to identify the next step that can be taken to transform the student's solution into a correct solution. The change between the student's solution and the next step is extracted and used to populate placeholders in a hint template to generate the hint presented to the student.

ASSISTments is an online learning system that teachers use to create problems with associated learning support (Heffernan \& Heffernan 2014, Razzaq et al. 2008). It supports the creation of problem, answer, and learning support templates, which content creators use to generate problem variations. Variables are input into the interface so they can be merged with the template to generate the problem and hint presented to the student.

Figure 3 shows a screenshot of ASSISTments' interface for creating problem templates. The problem uses placeholders for variables $a, b$, and answer, which are assigned the values specified in the Variables section. Figure 4 shows a hint template for the problem shown in Figure 3, which also uses variables $a, b$, and answer to generate the corresponding hints. Figure 5 shows an example of a problem and hint variation created using both templates.

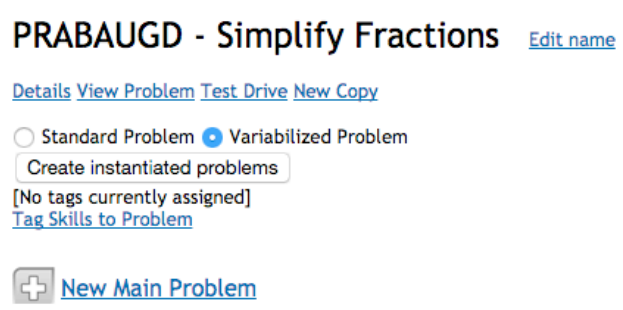

Main Problem 1
Hint strategy Hint
$\begin{aligned} & \text { * Click and drag main problems to re- } \\ & \text { order(may require refresh) }\end{aligned}$

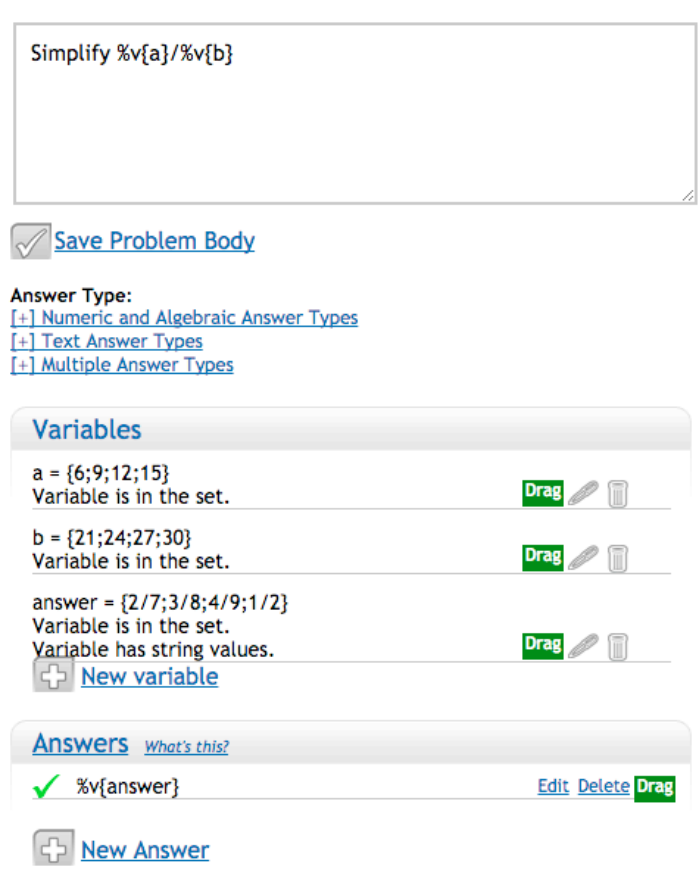

Fig. 3. Screenshot of an ASSISTments template used to generate problem variations and their corresponding answers (Image courtesy of ASSISTments http://assistments.org). 


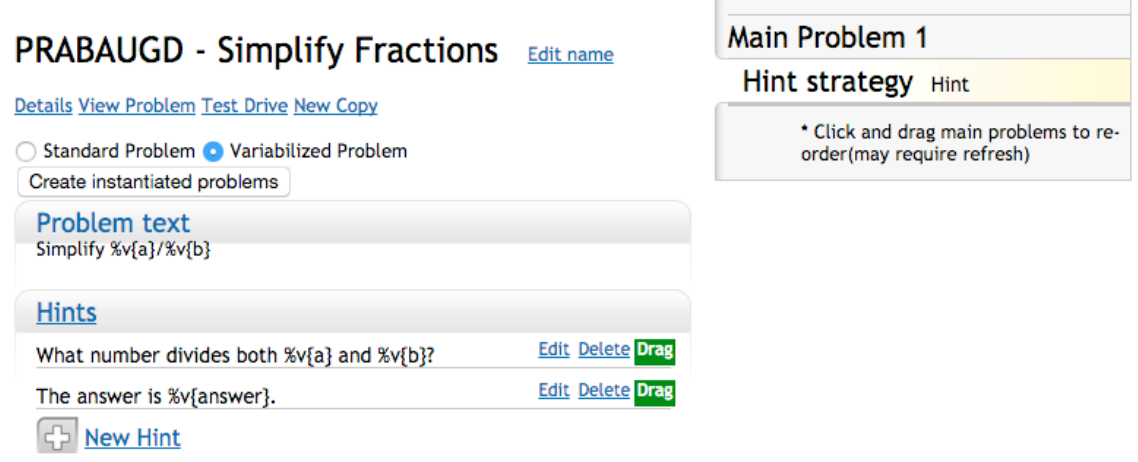

Fig. 4. Screenshot of an ASSISTments template used to generate corresponding hints for problem templates described in Figure 3 (Image courtesy of ASSISTments http://assistments.org).

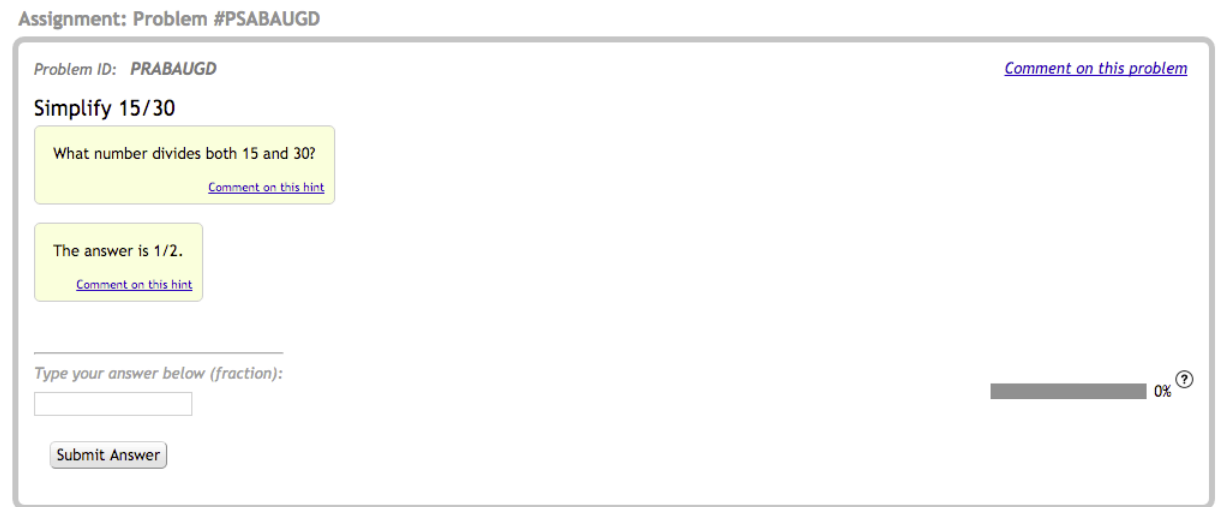

Fig. 5. Screenshot of an ASSISTments problem and its corresponding hints generated with the problem and hint templates shown in Figure 3 and Figure 4 (Image courtesy of ASSISTments http://assistments.org).

Related Patterns: The Mastery Learning Templates design pattern is used by the Mastery Learning Exercise Generator design pattern to generate exercises for mastery learning. Multiple hints may be associated with a single problem variation so students can request for more hints on a problem as described in the Increasing Hint Specificity design pattern. The automatic generation of exercises implements the Try It Yourself design pattern, and generating multiple variations of a problem implements the One Concept Several Implementations design pattern (Bergin et al. 2012). 


\section{Explain Worked Solutions}

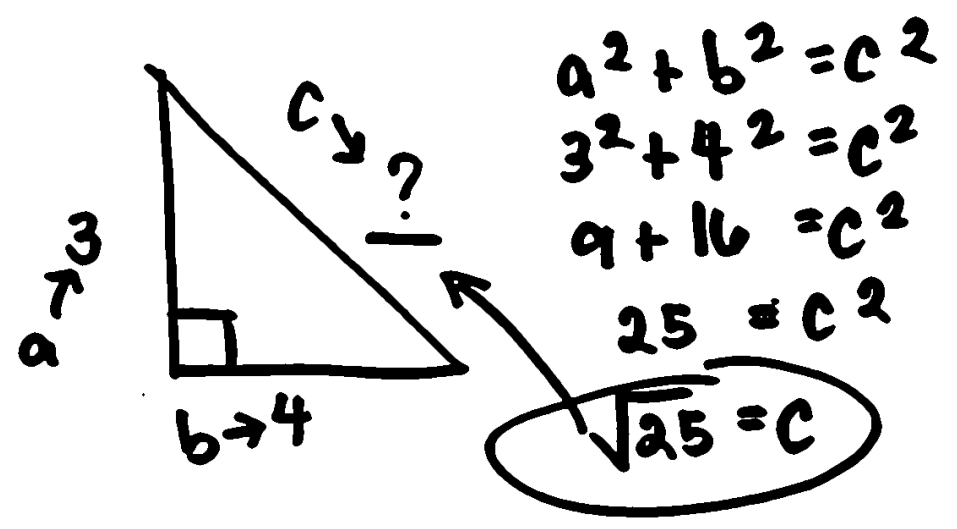

Context: Students are asked to answer problems in a math online learning system to practice a particular skill. The system provides students with different types of learning support to help them solve the problem such as hints generated using the Mastery Learning Templates and Mastery Learning Exercise Generator design patterns or guide questions built with the Scaffold Problems with Guide Questions design pattern.

Problem: Students may be unable to solve a problem even after making answer attempts and receiving learning support.

Forces:

1. Prior knowledge. Students cannot solve a problem if they lack the knowledge or skills necessary to solve it (Sweller 2004).

2. Learning opportunity. Students lose a learning opportunity if they are not taught how to solve a problem they failed to answer.

3. Disengagement. Students may disengage from the learning activity when they get stuck too long trying to solve a problem (D’Mello \& Graesser 2012).

Solution: Therefore, allow students to request for worked solutions if they tried to answer a problem and requested help, but were still unable to solve it. The worked solution should show each step in the solution process that starts with the given problem until it is solved. Each step should be explained clearly so students understand how the result of one step relates to the next step. Students may have a better chance of understanding the process and applying it to similar problems if they understand the process. Students may learn more from a worked solution because: (a) they are familiar with the problem (as they already tried to solve it), (b) they may be able to figure out what step they did incorrectly or did not perform, and (c) concise worked solutions guide student learning and do not introduce extraneous information that may cause additional cognitive load (Loibl \& Rummel 2014, Glogger-Frey et al. 2015). Students may feel a sense of accomplishment when they recognize the steps they performed in the worked solution. There may be more than one way to solve a problem, but it may be a good idea to present only one worked solution at a time to avoid confusion. Consider using the One Concept - Several Implementations design pattern (Bergin et al. 2012) by presenting different worked solutions for different variations of a problem so students become aware of different ways to solve it.

\section{Consequences:}

Benefits:

1. Worked solutions may help students acquire or relearn knowledge, which they can organize and store in long-term memory for later retrieval (Hume et al. 1996, Sweller 2004).

2. Providing clear explanations in the worked solution will help learners understand and apply what they learned to solve similar problems in the future (Wood et al. 1976, Mason \& Bruning 2001).

3. Worked solutions may help students get unstuck from the current problem. Understanding the solution may also help them avoid getting stuck on similar problems in the future. 
Liabilities:

1. Students may overlook critical information in the worked solution unless they are highlighted (Wood et al. 1976).

2. Content creators will need to create a worked solution for each problem they create.

3. Students may miss a learning opportunity when they ask for worked solutions too soon because the worked solution gives away the answer.

4. Students might not learn how to construct solutions on their own if they are used to having worked solutions.

5. Students may get the impression that a particular worked solution is the only way to solve the problem unless otherwise specified (e.g., explicitly stating there are other solutions, showing other solutions in other problems, asking students to compare their answers with their peers who might have used a different solution).

\section{Evidence:}

\section{Supporting Theories}

1. Learners need to recognize and understand the solution before they are able to apply it (Wood et al. 1976).

2. Several studies were conducted to compare the effects of various feedback types on learning such as no feedback, knowledge of response, knowledge of correct response, and elaborate feedback (Mason \& Bruning 2001). Many of these studies reported that elaborate feedback resulted in higher learning gains.

3. Worked solutions can help students recall previously acquired knowledge or help them acquire new knowledge that they can use to solve similar problems in the future (Hume et al. 1996, Sweller 2004).

4. Worked solutions can help students learn concepts effectively by reducing unnecessary cognitive load from extraneous information (Sweller 2006, Sweller et al. 2007).

5. Students can assess their knowledge-gaps more accurately when they try to solve problems before they receive instruction, which may also help prepare them for future learning (Loibl \& Rummel 2014, Glogger-Frey et al. 2015).

\section{Empirical Data}

Data was collected from the ASSISTments online learning system between September 2012 and September 2013. One of the features in the data set was students' average frustration, which described how likely students experienced frustration while answering a problem in ASSISTments. Student frustration was predicted by an affect detector using features like the number of previous incorrect answers, time taken to solve problems, and number of hint requests (Ocumpaugh et al. 2014). An analysis of frustration instances in the ASSISTments data set showed that some problems were more challenging than others based on the small number of students who answered it correctly. The analysis also revealed an interesting behavior wherein some students continually requested for hints until it showed them the solution and the correct answer, paused for a moment, submitted the correct answer, then moved on to the next problem. The next time these students encountered a similar problem, they answered it correctly on the first attempt. Students' usage of hints, when taken together, resembled a worked solution. This may indicate students' preference to learn from worked solutions, and its positive effects on their learning. More details about the data used, the methodology used for analysis, and the results are presented in (Inventado \& Scupelli 2015a).

\section{Known Implementations}

Many math textbooks and workbooks provide worked solutions in the back of the book so students can check their answers and see the correct solution. Several learning systems also provide students with worked solutions when they are unable to solve the problem such as ASSISTments and SQL-Tutor (Heffernan \& Heffernan 2014, Mitrovic 2012). ASSISTments, in particular, uses a variety of mediums to present worked solutions such as text and image explanations, video explanations, or short video lectures (Ostrow \& Heffernan 2014). 
Figure 6 illustrates a worked solution for a math problem about solving the sum of interior angles of triangles. The relevant concepts and the steps in the solution are explained so students can understand the process more easily. A better understanding of the problem and solution may enable them to apply this knowledge to similar problems.

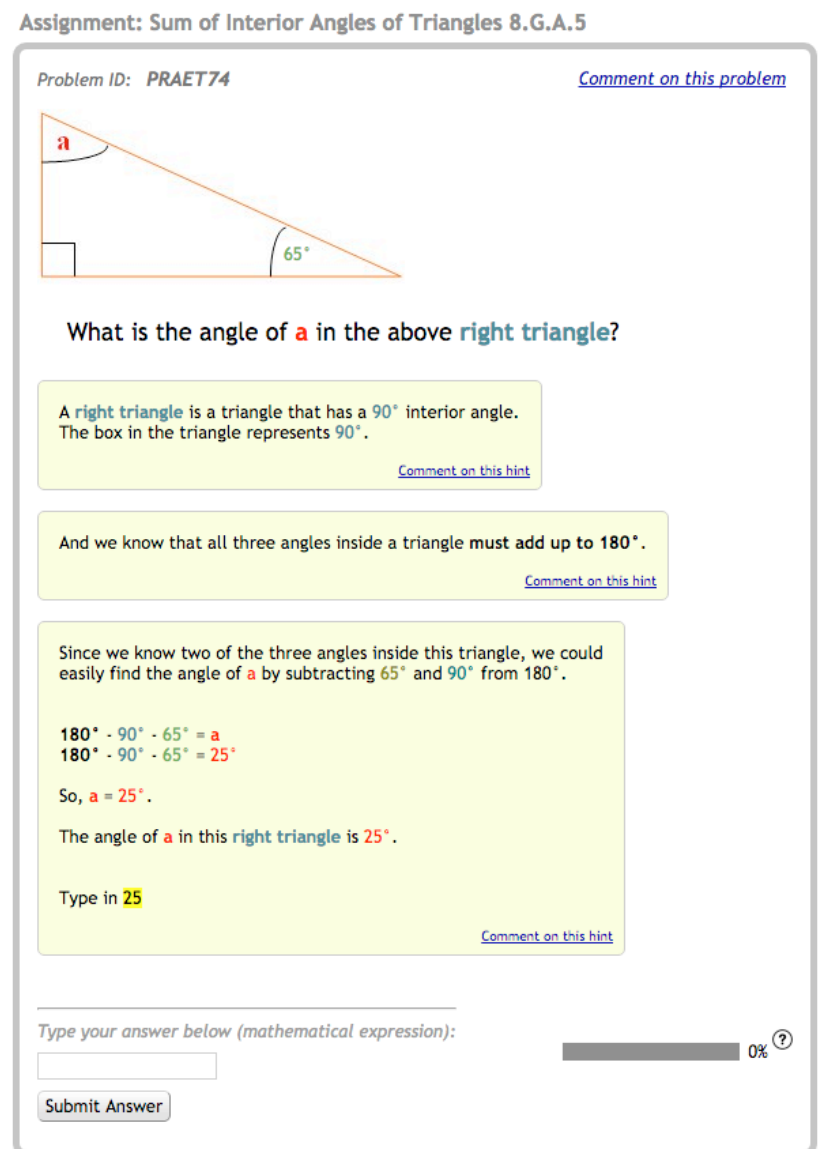

Fig. 6. Example of a worked solution in the ASSISTments online learning system (Image courtesy of ASSISTments http://assistments.org).

Related Patterns: The Explain Worked Solutions design pattern closely resembles the Worked Examples design pattern (Inventado \& Scupelli 2015b), but provides the answer to the current problem solved by the student. The Explain Worked Solutions design pattern can be used with the Try It Yourself design pattern (Bergin et al. 2012) to provide students with learning support while they answer problem-solving activities that promote better understanding of a given topic. Encoding worked solutions for problem variations may be tedious to write. Content creators may use the Mastery Learning Exercise Generator and Mastery Learning Templates design patterns to generate worked solutions automatically. It may be good idea to delay the presentation of worked solutions until students have attempted to solve the problem or tried to request basic hints as described in the Increasing Hint Specificity design pattern. The Image-Enhanced Hint design pattern (Inventado \& Scupelli 2016a) can be used to clarify worked solutions. If applicable, remember to implement the One Concept - Several Implementations design pattern (Bergin et al. 2012) to make students aware of other solutions that may solve the problem. 


\section{Scaffold Problems with Guide Questions}

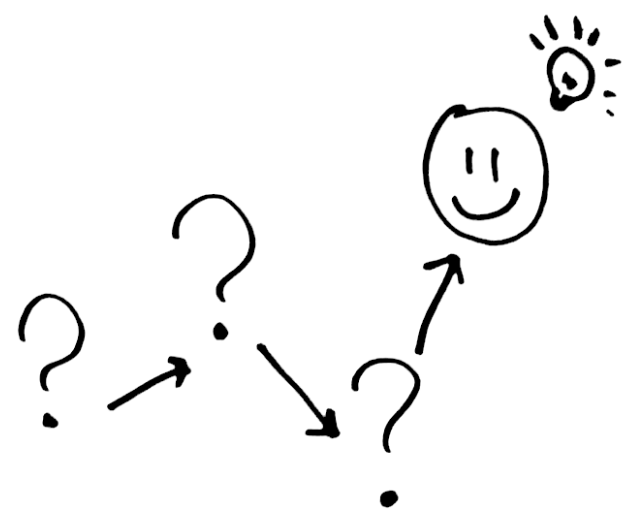

Context: Students are answering a multi-step math problem in an online learning system. By multi-step problems we mean math problems whose solution involves the application of a series of processes. For example, solving for the hypotenuse of a triangle requires multiple steps such as: (a) recall the Pythagorean equation, (b) substitute the value of the triangle's sides in the equation, (c) square the values of the sides, (d) add the squared values, and (e) get the square root of the sum.

Problem: Some students are unable to solve a problem because they may have forgotten or got confused about a concept or step in the solution process.

\section{Forces:}

1. Recalling prior knowledge. Students need to retrieve prior knowledge from long-term memory to utilize it in the working memory (Sweller et al. 1998). Various factors may hinder prior knowledge retrieval such as limited knowledge of related concepts, poor representation of the concept in memory, or lack of familiarity.

2. Availability bias. When students do not know how to solve a problem they may apply processes that they remember worked on previous problems (Sweller 2004). However, applying the wrong process on a problem and failing to solve it may affect students' motivation.

3. Limited working memory. The amount of information that can be held in memory while performing a task is limited (Sweller 2004). This makes it difficult to perform processes that consist of multiple concepts and steps.

Solution: Therefore, ask students to answer guide questions that may help them solve the problem. Guide questions can be used to help students recall information they need to solve the current step or recall the next step. For example, a multiple-choice question can help students recall the Pythagorean equation without necessarily giving away the answer. Guide questions may be asked to help students make inferences on the information they have available. For example, students could be asked how a given triangle's sides maps into the Pythagorean equation. The process of guiding students toward the solution is often called scaffolding because students are provided temporary support as they build their understanding of the concept. As students gain more experience solving the same problem type, students can be given less support (i.e., remove scaffolds) or given more complex problems such as what is described in the Personalized Problems design pattern (Inventado \& Scupelli 2015b).

\section{Consequences:}

\section{Benefits:}

1. Guide questions may help students retrieve relevant prior knowledge and focus their attention on important information so they can make inferences to solve the problem (Hume et al. 1996).

2. Students do not exert unnecessary time and effort trying out a solution that will not solve the problem.

3. Asking students to focus on a single concept at a time may minimize cognitive load and allow them to solve the problem more easily (Sweller 2004, Wood et al. 1976). 
Liabilities:

1. Content creators will need to create guide questions for each problem.

2. The system should be capable of controlling the amount of learning support provided to students over time so they are encouraged to solve problems on their own instead of depending on guide questions .

3. Students may misinterpret guide questions as extra work, which may discourage them from continuing to solve the problem.

4. Students who are only confused by one or a few steps need to go through all guide questions even if they already know the answers to most of those questions.

\section{Evidence:}

\section{Supporting Theories}

1. According to Vygotsky's Zone of Proximal Development (ZPD), providing students with guidance may help them accomplish tasks that they cannot do otherwise (Vygotsky 1987).

2. Students may find it difficult to solve problems that require too much cognitive load (Sweller 2004).

3. Human tutors often reduce the difficulty of a task to help students focus on one task, identify the correctness of their actions, and understand how to solve that task (Wood et al. 1976).

4. Guide questions may help students recall facts or make inferences that are necessary to solve the problem (Hume et al. 1996).

5. Scaffolding is an effective strategy for providing human and automated tutoring feedback (Chi et al. 2001, Merril et al. 1992, Wood et al. 1976).

\section{Empirical Data}

A randomized controlled trial (RCT) was conducted in ASSISTments to compare learning gains when students received learning support in the form of hints and scaffolds (Razzaq \& Heffernan 2006). Students in the hints condition received hints when they clicked on a hint request button. Students could request for one or more hints, wherein more specific information was revealed as they made hint requests. Students in the scaffold condition were asked to solve 2 to 4 guide questions whenever they could not answer the initial problem. The scaffolds were designed to help students perform a particular step in the solution process of the initial problem. The results of the experiment showed that students in the scaffold condition had higher learning gains compared to students in the hints condition.

\section{Known Implementations}

ASSISTments implements the Scaffold Problems with Guide Questions design pattern using what is referred to as a scaffold (Razzaq \& Heffernan 2006). Content creators construct an initial problem and optionally provide corresponding scaffolds for the problem. Students may request for scaffolds or are provided scaffolds automatically whenever they submit an incorrect answer. AutoTutor and DeepTutor are both learning systems that use conversational agents to help students learn through dialogue (Rus et al. 2013). Both systems rely heavily on scaffolding to help students recall the appropriate concepts to solve a given problem. Internally, these systems use natural language processing, latent semantic analysis, and production rules to produce guide questions based on student answers (Person et al. 2000). DeepTutor is an advanced version of AutoTutor that uses more advanced domain modeling methods, language processing, and tutorial strategies.

Figure 7 shows an example of a problem about the Pythagorean theorem (red border). When a student answers the problem incorrectly, the system provides the student with a scaffold (gray border). The scaffold asks students to identify the correct Pythagorean equation, which helps them recall the equation and prepares them for the next step in solution process. 


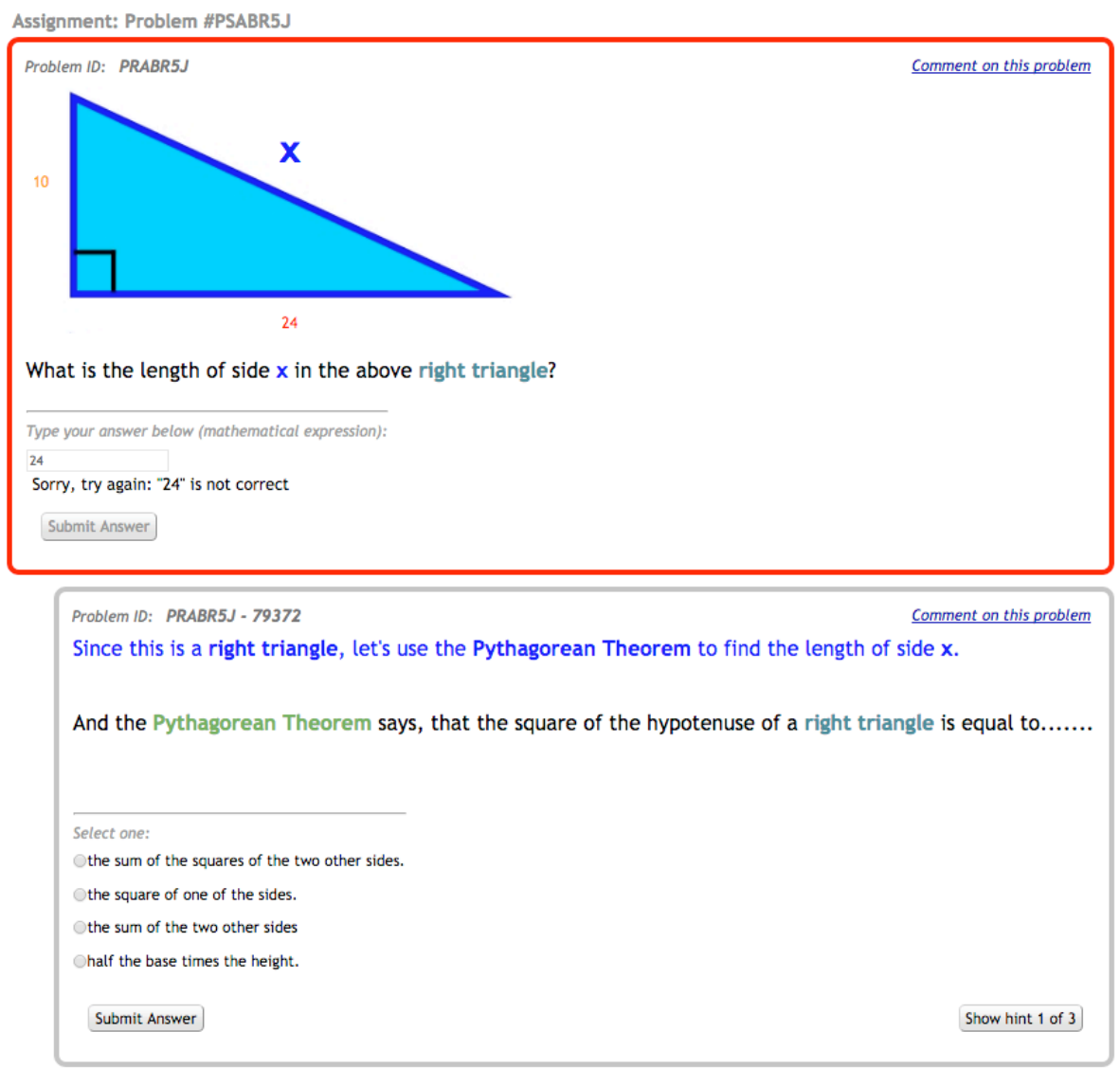

Fig. 7. A screenshot of a math problem in ASSISTments about the Pythagorean theorem (red border) and a scaffold (gray border) that was presented because the student answered the initial problem incorrectly (Image courtesy of ASSISTments http://assistments.org).

Related Patterns: The Scaffold Problems with Guide Questions pattern shares some ideas behind the Digestible Packets design pattern (Bergin et al. 2012) wherein the initial problem is broken into steps so students can concentrate on one problem at a time. Remember to implement the Keep It Simple design pattern (Cunningham \& Cunningham 2014) when constructing a scaffold to remove extraneous information and help students focus on one task. It is important to provide students with feedback as described in the Feedback design pattern. Hints may be provided to help students identify the answer. Worked solutions may be presented if the student is still unable to solve the problem as described in the Mastery Learning Template, Mastery Learning Exercise Generator, and Explain Worked Solutions design patterns. 


\section{SUMMARY AND NEXT STEPS}

The paper discussed three design patterns for constructing math-problem content and its associated learning support in online learning systems: Mastery Learning Templates, Explain Worked Solutions, and Scaffold Problems with Guide Questions. Online learning system developers, content creators, and teachers can use these patterns to guide the creation of problem content and learning support while ensuring its effectiveness in facilitating student learning.

The 3D2P methodology is currently being used on data collected from the ASSISTments online learning system to uncover more patterns that will be part of the Pattern Language for Math problems and Learning Support in Online Learning Systems. The design patterns are being compiled in an online design pattern repository (http://learningenvironmentslab.org/openpatternrepository) and work is being done to foster collaboration between design pattern authors, domain experts, and design pattern users to continue writing, evaluating, and refining design patterns.

\section{ACKNOWLEDGEMENTS}

This material is based upon work supported by the National Science Foundation under DRL-1252297. We would like to thank our shepherd Michael Weiss for his invaluable advice and feedback on this work. We would also like to thank Ryan Baker, Neil Heffernan, and their teams at Teachers College Columbia University and Worcester Polytechnic Institute for helping us analyze the data and gain insights on the methodology. We thank members of the Learning Environments Lab: Rachael Chang for her help with the randomized control trials and visualization of the 3D2P process; and Sharris Francisco-Inventado for her help with the visualization of the Pattern Language for Math problems and Learning Support in Online Learning Systems.

\section{REFERENCES}

Bergin, J., Eckstein, J., Völter, M., Sipos, M., Wallingford, E., Marquardt, K., Chandler, J., Sharp, H., and Manns, M.L. 2012. Pedagogical patterns: advice for educators. Joseph Bergin Software Tools.

Bjork, R.A. 1994. Memory and metamemory considerations in the training of human beings. In J. Metcalfe and A. Shimamura (Eds.), Metacognition: Knowing about knowing, Cambridge, MA: MIT Press, 185-205.

Bloom, B.S. 1968. Learning for Mastery. Instruction and Curriculum. Regional Education Laboratory for the Carolinas and Virginia, Topical Papers and Reprints, Number 1. Evaluation comment, 1(2), n2.

Cen, H., Koedinger, K. R., and Junker, B. 2007. Is Over Practice Necessary? Improving Learning Efficiency with the Cognitive Tutor through Educational Data Mining. Frontiers in Artificial Intelligence and Applications, 158, 511.

Chapanis, A., Garner, W. R., and Morgan, C. T. 1949. Applied experimental psychology: Human factors in engineering design. New York, NY: John Wiley \& Sons.

Chi, M. T., Siler, S. A., Jeong, H., Yamauchi, T., and Hausmann, R. G. 2001. Learning from human tutoring. Cognitive Science, 25(4), $471-533$.

Cox Jr, W.F., and Dunn, T.G. 1979. Mastery learning: A psychological trap? Educational Psychologist, 14(1), 24-29.

Cunningham and Cunningham Inc. 2014. Keep it Simple. Retrieved from http://c2.com/cgi/wiki?KeepItSimple.

edX - Free Online Courses from the World's Best Universities .n.d. Retrieved December 17, 2015 from http://www.edx.org

D’Mello, S., and Graesser, A. 2012. Dynamics of affective states during complex learning. Learning and Instruction, 22(2), $145-157$.

Glogger-Frey, I., Fleischer, C., Grueny, L., Kappich, J., and Renkl, A. 2015. Inventing a solution and studying a worked solution prepare differently for learning from direct instruction. Learning and Instruction, 39, 72-87.

Guskey, T.R. 2008. Mastery Learning. In Good, T.L. (Ed.), 21st Century Education: A Reference Handbook, Thousand Oak, CA: Sage Publicaitons, I-254.

Guskey, T.R., and Gates, S.L. 1985. A Synthesis of Research on Group-Based Mastery Learning Programs. Paper presented at 69th the Annual Meeting of the American Educational Research Association, 66 pages.

Head, J., and Helton, W. S. 2014. Sustained attention failures are primarily due to sustained cognitive load not task monotony. Acta psychologica, 153, 87-94.

Heffernan, N. T., and Heffernan, C. L. 2014. The ASSISTments Ecosystem: Building a platform that brings scientists and teachers together for minimally invasive research on human learning and teaching. International Journal of Artificial Intelligence in Education, 24(4), 470497.

Hume, G., Michael, J., Rovick, A., and Evens, M. 1996. Hinting as a tactic in one-on-one tutoring. The Journal of the Learning Sciences, 5(1), 23-47.

Inventado, P.S. and Scupelli, P. 2015a. Data-driven design pattern production: a case study on the ASSISTments online learning system. In Proceedings of the 20th European Conference on Pattern Languages of Programs (EuroPLoP '15). ACM, New York, NY, USA, Article 14, 13 pages.

Inventado, P.S. and Scupelli, P. 2015b. A Data-driven Methodology for Producing Online Learning System Design Patterns. In Proceedings of the 22nd Conference on Pattern Languages of Programs (PLoP '15). ACM, New York, NY, USA.

Inventado, P.S. and Scupelli, P. 2016a. Design Patterns for Helping Students to Learn to Visualize Math Problems in Online Learning Systems. In Proceedings of the 21st European Conference on Pattern Languages of Programs (EuroPLoP '16).

Inventado, P.S. and Scupelli, P. 2016b. Learning-Support Design Patterns for Student Interactions with Math Problems. In Proceedings of the 23rd Conference on Pattern Languages of Programs (PLoP'16). 
Inventado, P.S., Scupelli, P., VanInwegen, E., Ostrow, K., Heffernan, N., Ocumpaugh, J., Baker, R., Slater, S., and Almeda, V. 2016. Hint Availability Slows Completion Times in Summer Work. In Tiffany Barnes, Min Chi and Mingyu Feng (eds.) Proceedings of the 9th International Conference on Educational Data Mining.

Koedinger, K. R., and Aleven, V. 2007. Exploring the assistance dilemma in experiments with cognitive tutors. Educational Psychology Review, 19(3), 239-264.

Köppe, C., Niels, R., Holwerda, R., Tijsma, L., van Diepen, N., van Turnhout, K., Bakker, R. 2015. Flipped Classroom Patterns - Designing Valuable In-Class Meetings. Proceedings of the 20th European Conference on Pattern Languages of Programs, EuroPLoP'15. Irsee, Germany

Kulik, C.L.C., Kulik, J.A., and Bangert-Drowns, R.L. 1990. Effectiveness of mastery learning programs: A meta-analysis. Review of educational research, 60(2), 265-299.

Loibl, K., and Rummel, N. 2014. Knowing what you don't know makes failure productive. Learning and Instruction, 34, 74-85.

Mason, B. J., and Bruning, R. 2001. Providing feedback in computer-based instruction: What the research tells us. Center for Instructional Innovation, University of Nebraska-Lincoln: 14. Retrieved January 3, 2015, from http://dwb.unl.edu/Edit/MB/MasonBruning.html

Merrill, D. C., Reiser, B. J., Ranney, M., and Trafton, J. G. 1992. Effective tutoring techniques: A comparison of human tutors and intelligent tutoring systems. The Journal of the Learning Sciences, 2(3), 277-305.

Mitrovic, A. 2012. Fifteen years of constraint-based tutors: what we have achieved and where we are going. User Modeling and UserAdapted Interaction, 22(1-2), 39-72.

Mor, Y., and Warburton, S. 2015. Practical Patterns for Active and Collaborative MO0Cs: Checkpoints, FishBowl and See Do Share. eLearning, 48:48-56.

Ocumpaugh, J., Baker, R., Gowda, S., Heffernan, N., \& Heffernan, C. 2014. Population validity for Educational Data Mining models: A case study in affect detection. British Journal of Educational Technology, 45(3), 487-501.

Ostrow, K. S., and Heffernan, N. T. 2014. Testing the multimedia principle in the real world: a comparison of video vs. Text feedback in authentic middle school math assignments. In Proceedings of the 7th Int Conf on EDM (pp. 296-299).

Person, N., Graesser, A.C., and the Tutoring Research Group. 2000. Designing AutoTutor to be an Effective Conversational Partner. In B. Fishman \& S. O'Connor-Divelbiss (Eds.), Fourth International Conference of the Learning Sciences (pp. 246-253). Mahwah, NJ: Erlbaum.

Razzaq, L., and Heffernan, N. T. 2006. Scaffolding vs. hints in the Assistment System. In Intelligent Tutoring Systems (pp. 635-644). Springer Berlin Heidelberg.

Razzaq, L., Patvarczki, J., Almeida, S., Vartak, M., Feng, M., Heffernan, N. T., and Koedinger, K. R. (2008). The ASSISTment Builder: Supporting the life cycle of ITS content creation (WPI Tech Report No. WPI-CS-TR-08-06). Worcester, MA: Worcester Polytechnic Institute.

Rivers, K., and Koedinger, K. R. 2015. Data-Driven Hint Generation in Vast Solution Spaces: a Self-Improving Python Programming Tutor. International Journal of Artificial Intelligence in Education, 1-28.

Rus, V., D'Mello, S., Hu, X., and Graesser, A. 2013. Recent advances in conversational intelligent tutoring systems. AI magazine, 34 (3), $42-54$.

Salden, R. J., Aleven, V., Schwonke, R., and Renkl, A. 2010. The expertise reversal effect and worked examples in tutored problem solving. Instructional Science, 38(3), 289-307.

Slavin, R.E. 1987. Mastery learning reconsidered. Review of educational research, 57(2), 175-213.

Sweller, J. 2004. Instructional design consequences of an analogy between evolution by natural selection and human cognitive architecture. Instructional science, 32(1-2), 9-31.

Sweller, J. 2006. The worked example effect and human cognition. Learning and Instruction, 16(2), 165-169.

Sweller, J., Kirschner, P. A., and Clark, R. E. 2007. Why minimally guided teaching techniques do not work: A reply to commentaries. Educational Psychologist, 42(2), 115-121.

Sweller, J., Van Merrienboer, J. J., and Paas, F. G. 1998. Cognitive architecture and instructional design. Educational psychology review, 10(3), 251-296.

U.S. Department of Education, Institute of Education Sciences, What Works Clearinghouse. 2009. Cognitive Tutor ${ }^{\circledR}$ Algebra I. Retrieved December 16, 2015 from http://whatworks.ed.gov

Vygotsky, L. 1987. Zone of proximal development. Mind in society: The development of higher psychological processes, 5291.

Warburton, S., and Mor, Y. 2015. A set of patterns for the structured design of MOOCs. Open Learning: The Journal of Open, Distance and eLearning, 1-15.

Wickens, C. D., Lee, J., Liu, Y., and Becker, S. G. 2004. An introduction to human factors engineering.

Wood, D., Bruner, J. S., and Ross, G. 1976. The role of tutoring in problem solving. Journal of child psychology and psychiatry, 17(2), 89-100. 